\title{
On Stability in Fuzzy Linear Programming Problems *
}

\author{
Robert Fullér \\ rfuller@abo.fi
}

\begin{abstract}
This study focuses on the problem of stability (with respect to changes of centres of fuzzy parameters) of the solution in Fuzzy Linear Programming (FLP) problems with symmetrical triangular fuzzy numbers and extended operations and inequalities.
\end{abstract}

\section{Introduction}

Sensitivity analysis in FLP problems (with crisp parameters and soft constraints) was first considered in [2], where a functional relationship between changes of parameters of the right-hand side and those of the optimal value of the primal objective function was derived for almost all conceivable cases.

In [3] a FLP problem (with symmetrical triangular fuzzy numbers) was formulated and the value of information was discussed via sensitivity analysis. In the present paper we investigate the stability of the solution in FLP problems (with symmetrical triangular fuzzy numbers and extended operations and inequalities) with respect to changes of fuzzy parameters. We show that the solution to these problems is stable (in metric C) under variations in the membership function of the fuzzy coefficients.

\section{Preliminaries}

Definition 2.1. A fuzzy set of the real line given by the membership function

$$
\tilde{a}(t)= \begin{cases}1-\frac{|a-t|}{\alpha} & \text { if }|a-t| \leq \alpha \\ 0 & \text { otherwise }\end{cases}
$$

Where $\alpha>0$ will be called a symmetrical triangular fuzzy number with center $a \in \mathbb{R}$ and width $2 \alpha$ and we shall refer to it by the pair $(a, \alpha)$.

Let $\tilde{a}=(a, \alpha)$ and $\tilde{b}=\left(b, \alpha^{\prime}\right)$ be two fuzzy numbers of the form (1), $\lambda \in \mathbb{R}$. Then it is easily verified from Zadeh's extension principle that we have

$$
\tilde{a}+\tilde{b}=\left(a+b, \alpha+\alpha^{\prime}\right), \quad \tilde{a}-\tilde{b}=\left(a-b, \alpha+\alpha^{\prime}\right), \quad \lambda \tilde{a}=(\lambda a,|\lambda| \alpha) .
$$

\footnotetext{
${ }^{*}$ The final version of this paper appeared in R. Fullér, On Stability in Fuzzy Linear Programming Problems, Fuzzy Sets and Systems, 30(1989) 339-344. doi: 10.1016/0165-0114(89)90026-2
} 
The degree of satisfaction of the assertion " $\tilde{a}$ is less than $\tilde{b}$ ", which we write $\tilde{a} \lesssim \tilde{b}$, is $v(\tilde{a}, \tilde{b})$ defined in [1] as

$$
v(\tilde{a}, \tilde{b})=\sup _{x \leq y} \tilde{a}(x) \wedge \tilde{b}(y) .
$$

This formula uses once more Zadeh's extension principle.

Let $\tilde{a}=(a, \alpha)$ and $b=\left(b, \alpha^{\prime}\right)$ be two fuzzy numbers of the form (1). Then it can be easily checked that

$$
v(\tilde{a}, \tilde{b})= \begin{cases}1 & \text { if } a \leq b, \\ 1-\frac{a-b}{\alpha+\alpha^{\prime}} & \text { if } b<a \leq b+\alpha+\alpha^{\prime}, \\ 0 & \text { if } a>b+\alpha+\alpha^{\prime} .\end{cases}
$$

\section{Stability of the solution for the FLP problem}

The conventional model of LP can be stated as

$$
\left\langle a_{0}, x\right\rangle \rightarrow \min ; \text { subject to } A x \leq b .
$$

In many real-world problems instead of minimization of the objective function (5) it may be sufficient to determine an $x$ such that

$$
\begin{gathered}
a_{01} x_{1}+\cdots+a_{0 n} x_{n} \leq b_{0}, \\
a_{11} x_{1}+\cdots+a_{1 n} x_{n} \leq b_{1}, \\
\vdots \\
a_{m 1} x_{1}+\cdots+a_{m n} x_{n} \leq b_{m}
\end{gathered}
$$

where $b_{0}$ is a predetermined aspiration level.

Assume that all parameters in (6) are fuzzy and are described by symmetrical triangular fuzzy numbers. Then the following FLP problem can be obtained by replacing crisp parameters $a_{i j}, b_{i}$ with fuzzy numbers $(1) \tilde{a}_{i j}=\left(a_{i j}, \alpha\right), \tilde{b}_{i}=\left(b_{i}, d_{i}\right)$ respectively and by replacing crisp relation $\leq$ with extended relation $\lesssim$.

$$
\left(a_{i 1}, \alpha\right) x_{1}+\cdots+\left(a_{i n}, \alpha\right) x_{n} \lesssim\left(b_{i}, d_{i}\right), \quad i=0, \ldots, m .
$$

We denote by $\mu_{i}(x)$ the degree of satisfaction of the $i$-th restriction at the point $x \in \mathbb{R}^{n}$ in (7), i.e. $\mu_{i}(x)=v\left(\tilde{a}_{i 1} x_{1}+\cdots+\tilde{a}_{i n} x_{n}, \tilde{b}_{i}\right)$.

Then the solution of the FLP problem (7) is defined as a fuzzy set on $\mathbb{R}^{n}$ whose membership function is given by

$$
\mu(x)=\min _{i=0, \ldots, m} \mu_{i}(x)
$$

and the maximizing solution $x^{*}$ of the FLP problem (7) satisfies the equation

$$
\mu\left(x^{*}\right)=\mu^{*}=\max _{x \in \mathbb{R}^{n}} \mu(x) .
$$

From (2) and (4) it follows that the degree of satisfaction of the $i$-th restriction at the point $x \in \mathbb{R}^{n}$ in (7) is the following: 


$$
\mu_{i}(x)= \begin{cases}1 & \text { if }\left\langle a_{i}, x\right\rangle \leq b_{i}, \\ 1-\frac{\left\langle a_{i}, x\right\rangle-b_{i}}{\alpha|x|_{1}+d_{i}} & \text { otherwise, } \\ 0 & \text { if }\left\langle a_{i}, x\right\rangle>b_{i}+\alpha|x|_{1}+d_{i}\end{cases}
$$

where $|x|_{1}=\left|x_{1}\right|+\cdots+\left|x_{n}\right|$ and $\left\langle a_{i}, x\right\rangle=a_{i 1} x_{1}+\cdots+a_{i n} x_{n}, i=0,1, \ldots, m$.

Remark 3.1. In the extremal case $\alpha=0$ but $d_{i}>0$ in (10), we get a linear membership function, i.e. Zimmermann's principle [4].

Assume that instead of exact centers $a_{i j}, b_{i}$ in (7) only their approximations $a_{i j}(\delta), b_{i}(\delta)$ are known such that

$$
\max _{i, j}\left|a_{i j}-a_{i j}(\delta)\right| \leq \delta, \quad \max _{i}\left|b_{i}-b_{i}(\delta)\right| \leq \delta,
$$

Then we get the following FLP problem:

$$
\left(a_{i 1}(\delta), \alpha\right) x_{1}+\cdots+\left(a_{i n}(\delta), \alpha\right) x_{n} \lesssim\left(b_{i}(\delta), d_{i}\right), \quad i=0, \ldots, m .
$$

In a similar manner we can define the solution of the FLP problem (12) by

$$
\mu^{\delta}(x)=\min _{i=0, \ldots, m} \mu_{i}^{\delta}(x), x \in \mathbb{R}^{n}
$$

where $\mu_{i}^{\delta}(x)$ is the degree of satisfaction of the $i$-th restriction at the point $x \in \mathbb{R}^{n}$ in (12) and the maximizing solution $x^{*}(\delta)$ of the FLP problem (12) satisfies the equation

$$
\mu^{\delta}\left(x^{*}(\delta)\right)=\mu^{*}(\delta)=\sup _{x \in \mathbb{R}^{n}} \mu^{\delta}(x) .
$$

In the following theorem we establish a stability of the solution of the FLP problem (7).

Theorem 3.1. Let $\mu(x)$ and $\mu^{\delta}(x)$ be solution of FLP problems (7) and (12) respectively. Then

$$
\left\|\mu-\mu^{\delta}\right\|_{C}=\sup _{x \in \mathbb{R}^{n}}\left|\mu(x)-\mu^{\delta}(x)\right| \leq \delta\left(\frac{1}{\alpha}+\frac{1}{d}\right)
$$

where $d=\min \left\{d_{0}, d_{1}, \ldots, d_{m}\right\}$.

Proof. First let $\delta \geq \min \{\alpha, d\}$. Then from $\left|\mu(x)-\mu^{\delta}(x)\right| \leq 1, \forall x \in \mathbb{R}^{n}$ and $\delta\left(\alpha^{-1}+d^{-1}\right) \geq 1$ we obtain (14).

Suppose that

$$
0<\delta<\min \{\alpha, d\}
$$

It will be sufficient to show that

$$
\left|\mu_{i}(x)-\mu_{i}^{\delta}(x)\right| \leq \delta\left(\frac{1}{\alpha}+\frac{1}{d}\right), \forall x \in \mathbb{R}^{n}, \quad i=0, \ldots, m,
$$

because from (16) follows (14).

Let $x \in \mathbb{R}^{n}$ and $i \in\{0, \ldots, m\}$ be arbitrarily fixed. Consider the following cases: 
(1) $\mu_{i}(x)=\mu_{i}^{\delta}(x)$. In this case (16) is trivially obtained.

(2) $0<\mu_{i}(x)<1$ and $0<\mu_{i}^{\delta}(x)<1$. In this case from (10), (11) we have

$$
\begin{gathered}
\left|\mu_{i}(x)-\mu_{i}^{\delta}(x)\right|= \\
\left|1-\frac{\left\langle a_{i}, x\right\rangle-b_{i}}{\alpha|x|_{1}+d_{i}}-\left[1-\frac{\left\langle a_{i}(\delta), x\right\rangle-b_{i}(\delta)}{\alpha|x|_{1}+d_{i}}\right]\right|= \\
\frac{\left|b_{i}-b_{i}(\delta)+\left\langle a_{i}(\delta), x\right\rangle-\left\langle a_{i}, x\right\rangle\right|}{\alpha|x|_{1}+d_{i}} \leq \\
\frac{\left|b_{i}-b_{i}(\delta)+\right|\left\langle a_{i}(\delta),-a_{i}, x\right\rangle \mid}{\alpha|x|_{1}+d_{i}} \leq \frac{\delta+\left|a_{i}(\delta)-a_{i}\right|_{\infty}|x|_{1}}{\alpha|x|_{1}+d_{i}} \leq \\
\frac{\delta+\delta|x|_{1}}{\alpha|x|_{1}+d_{i}} \leq \delta\left(\frac{1}{\alpha}+\frac{1}{d_{i}}\right) \leq \delta\left(\frac{1}{\alpha}+\frac{1}{d}\right)
\end{gathered}
$$

where $a_{i}(\delta)=\left(a_{i 1}(\delta), \ldots, a_{i n}(\delta)\right)$ and $\left|a_{i}(\delta)-a_{i}\right|_{\infty}=\max _{j}\left|a_{i j}(\delta)-a_{i j}\right|$.

(3) $\mu_{i}(x)=1$ and $0<\mu_{i}^{\delta}(x)<1$. In this case from (10) we have

$$
\left\langle a_{i}, x\right\rangle \leq b_{i}
$$

Hence

$$
\begin{gathered}
\left|\mu_{i}(x)-\mu_{i}^{\delta}(x)\right|=\left|1-\left[1-\frac{\left\langle a_{i}(\delta), x\right\rangle-b_{i}(\delta)}{\alpha|x|_{1}+d_{i}}\right]\right|= \\
\frac{\left\langle a_{i}(\delta), x\right\rangle-b_{i}(\delta)}{\alpha|x|_{1}+d_{i}} \leq \\
\frac{\left\langle a_{i}(\delta), x\right\rangle-b_{i}(\delta)-\left(\left\langle a_{i}, x\right\rangle-b_{i}\right)}{\alpha|x|_{1}+d_{i}} \leq \delta\left(\frac{1}{\alpha}+\frac{1}{d}\right) .
\end{gathered}
$$

(4) $0<\mu_{i}(x)<1$ and $\mu_{i}^{\delta}(x)=1$. In this case the proof is carried out analogously to the proof of the preceding case.

(5) $0<\mu_{i}(x)<1$ and $\mu_{i}^{\delta}(x)=0$. In this case from

$$
\left\langle a_{i}(\delta), x\right\rangle-b_{i}(\delta)>\alpha|x|_{1}+d_{i}
$$

it follows that

$$
\begin{gathered}
\left|\mu_{i}(x)-\mu_{i}^{\delta}(x)\right|=\left|1-\frac{\left\langle a_{i}, x\right\rangle-b_{i}}{\alpha|x|_{1}+d_{i}}\right|= \\
\left.\frac{1}{\alpha|x|_{1}+d_{i}}|\alpha| x\right|_{1}+d_{i}-\left(\left\langle a_{i}, x\right\rangle-b_{i}\right) \mid \leq \\
\frac{\left|\left\langle a_{i}(\delta), x\right\rangle-b_{i}(\delta)-\left(\left\langle a_{i}, x\right\rangle-b_{i}\right)\right|}{\alpha|x|_{1}+d_{i}} \leq \delta\left(\frac{1}{\alpha}+\frac{1}{d}\right) .
\end{gathered}
$$


(6) $\mu_{i}(x)=0$ and $0<\mu_{i}^{\delta}(x)<1$. In this case the proof is carried out analogously to the proof of the preceding case.

(7) $\mu_{i}(x)=1 \mu_{i}^{\delta}(x)=0$, or $\mu_{i}(x)=0, \mu_{i}^{\delta}(x)=1$. These cases are not reasonable. For instance suppose that case $\mu_{i}(x)=1, \mu_{i}^{\delta}(x)=0$ is conceivable. Then from (11) it follows that

$$
\begin{gathered}
\left|\left\langle a_{i}, x\right\rangle-b_{i}-\left(\left\langle a_{i}(\delta), x\right\rangle-b_{i}(\delta)\right)\right| \leq \\
\left|b_{i}-b_{i}(\delta)\right|+\left|a_{i}(\delta)-a_{i}\right|_{\infty}|x|_{1} \leq \delta\left(|x|_{1}+1\right) .
\end{gathered}
$$

On the other hand from (15), (17), (18) we have

$$
\begin{gathered}
\left|\left\langle a_{i}, x\right\rangle-b_{i}-\left(\left\langle a_{i}(\delta), x\right\rangle-b_{i}(\delta)\right)\right| \geq \\
\left|\left\langle a_{i}(\delta), x\right\rangle-b_{i}(\delta)\right| \geq \alpha|x|_{1}+d_{i}>\delta|x|_{1}+\delta=\delta\left(|x|_{1}+1\right) .
\end{gathered}
$$

But these form a contradiction, which ends the proof.

Remark 3.2. From (14) it follows that

$$
\left\|\mu-\mu^{\delta}\right\|_{C} \rightarrow 0 \quad \text { if } \delta / \alpha \rightarrow 0 \text { and } \delta / d \rightarrow 0,
$$

which means stability with respect to perturbations (11) of the solution in FLP problem (7).

Remark 3.3. It is easily verified that problem (9) is equivalent to

$$
\begin{aligned}
& \max \lambda \\
& \lambda\left(\alpha|x|_{1}+d_{i}\right)-\alpha|x|_{1}+\left\langle a_{i}, x\right\rangle \leq b_{i}+d_{i}, \quad i=0, \ldots, m, \\
& 0 \leq \lambda \leq 1, \quad x \in \mathbb{R}^{n} .
\end{aligned}
$$

The resulting problem is a nonlinear programming one.

Remark 3.4. From 14 it follows that

$$
\left|\mu^{*}-\mu^{*}(\delta)\right| \leq \delta\left(\frac{1}{\alpha}+\frac{1}{d}\right)
$$

where $\mu^{*}, \mu^{*}(\delta)$ are the optimal values of the objective functions in problems (9) and (13) respectively.

Remark 3.5. It is easily checked that in the extremal case $\alpha=0$ but $d_{i}>0$, the solution of the FLP problem (7) may be unstable with respect to changes of the crisp parameters $a_{i j}, b_{i}$.

\section{Concluding remarks}

In this paper we have shown that the solution of the FLP problem (7) has a stability property with respect to changes of centres of fuzzy parameters. From (20) it follows that the optimal value of the objective function in the problem (19) has a stability propertu, but the stability or instability of the maximizing solution is an open problem yet, i.e. the problem: will the following statement be true?

$$
\rho\left(x^{*}(\delta), X^{*}\right) \rightarrow 0 \text { if } \delta \rightarrow 0,
$$

where $X^{*} \neq \emptyset$ denotes the set of maximizing solutions of the problem (7), $x^{*}(\delta)$ is a maximizing solution of the problem (13) and $\rho$ is a metric in $\mathbb{R}^{n}$. 


\section{Acknowledgments}

The author would like to express his gratitude to Dr. Fedor Pavlovich Vasil'ev of Moscow State University and to Dr. Kovács Margit of the Computing Center of the Eötvös Loránd University, Budapest for their helpful discussion.

\section{References}

[1] D. Dubois and H. Prade, System of linear fuzzy constraints, Fuzzy Sets and Systems 3(1980) $37-48$.

[2] H. Hamacher, H. Leberling and H. -J. Zimmermann, Sensitivity analysis in fuzzy linear programming, Fuzzy Sets and Systems 1(1978) 269-281.

[3] H. Tanaka, H. Ichihashi and K. Asai, A value of information in FLP problems via sensitivity analysis, Fuzzy Sets and Systems 18(1986) 119-129.

[4] H. -J. Zimmermann, Description and optimization of fuzzy systems, Internat. J. General Systems 2(1975) 209-215.

\section{Extensions}

The results of this paper were extended to FLP with continuous fuzzy number coefficients in:

- M.Fedrizzi and R.Fullér, Stability in possibilistic linear programming problems with continuous fuzzy number parameters, Fuzzy Sets and Systems, 47(1992) 187-191.

and to FMOP with continuous fuzzy coefficients in:

- R.Fullér and M.Fedrizzi, On stability in multiobjective possibilistic linear programs, European Journal of Operational Research, 74(1994) 179-187.

\section{Follow ups}

The results of this paper have been used later in the following works.

\section{in journals}

A35-c21 B Kheirfam, F Hasani, Sensitivity analysis for fuzzy linear programming problems with fuzzy variables, ADVANCED MODELING AND OPTIMIZATION, 12(2010), number 2, pp. 257-272. 2010

http://camo.ici.ro/journal/vol12/v12b11.pdf 
Fullér [A35] showed that the solution to FLP problems with symmetrical triangular fuzzy numbers is stable with respect to small changes of centers of fuzzy numbers. Perturbations occur due to calculation errors or just to answer managerial questions "What if ...". Such questions propose after the simplex method and the related research area refers to as basis invariancy sensitivity analysis. (page 258)

A35-c20 Wen-Pai Wang, An approach to initiating product design in mass customisation environments, INTERNATIONAL JOURNAL OF LOGISTICS SYSTEMS AND MANAGEMENT, vol. 3, pp. 330-343. 2007

http://dx.doi.org/10.1504/IJLSM.2007.012997

A35-c19 Rasoul Dadashzadeh; S. B. Nimse, ON STABILITY IN MULTIOBJECTIVE LINEAR PROGRAMMING PROBLEMS WITH SYMMETRIC TRAPEZOIDAL FUZZY NUMBERS, ANNALS OF ORADEA UNIVERSITY - MATHEMATICS FASCICOLA, Tom XIV(2007), pp. 514. 2007

http://stiinte.uoradea.ro/en/pdfs/2007/1.pdf

Abstract. In 1989 Fullér revealed the stability in fuzzy linear programming problems (FLP) with symmetric triangular fuzzy numbers. Also Dadashzadeh et. al., showed the stability in fuzzy linear programming with symmetric trapezoidal fuzzy numbers. Connected to the above mentioned matters, in this paper we study the stability of solution in fuzzy multiobjective linear programming (FMLP) problems with symmetric trapezoidal fuzzy numbers, with respect to the change of fuzzy parameters. (page 5)

A35-c18 Pankaj Gupta and Davinder Bhatia, Sensitivity analysis in fuzzy multiobjective linear fractional programming problem, FUZZY SETS AND SYSTEMS, 122(2001) 229-236. 2001 http://dx.doi.org/10.1016/S0165-0114(99)00164-5

Sensitivity analysis in FLP problems with crisp parameters and soft constraints was considered first by Hamacher et al. [8] and later by many others, e.g. Tanaka et al. [16], and Fullér [A35].

A35-c17 Rybkin VA, Yazenin AV, On the problem of stability in possibilistic optimization INT J GEN SYST 30 (1): 3-22 2001

http://dx.doi.org/10.1080/03081070108960695

A35-c16 Ammar EE, Kassem MAEH, On stability analysis of multicriteria LP problems with fuzzy parameters FUZZY SET SYST 82 (3): 331-334 SEP 231996

http://dx.doi.org/10.1016/0165-0114(95)00266-9

A35-c15 Julien B, Water-quality management with imprecise information, EUROPEAN JOURNAL OF OPERATIONAL RESEARCH, 76 (1): 15-27, JUL 61994

http://dx.doi.org/10.1016/0377-2217(94)90003-5

A35-c14 M.Delgado, F.Herrera, J.L.Verdegay and M.A.Vila, Post-optimality analysis on the membership functions of a fuzzy linear programming problem, FUZZY SETS AND SYSTEMS, 53(1993) 289-297. 1993

http://dx.doi.org/10.1016/0165-0114(93)90400-C 
A35-c13 D.Dutta, J.R.Rao and R.N. Tiwari, Effect of tolerance in fuzzy linear fractional programming, FUZZY SETS AND SYSTEMS, 55(1993) 133-142. 2003

Fullér [A35] has studied the problem of stability with respect to changes of centres of fuzzy parameters of the solution in FLP problems with symmetrical triangular fuzzy numbers and extended operations and inequalities. (page 134)

A35-c12 M.Kovács, Stable embeddings of linear equality and inequality systems into fuzzified systems, FUZZY SETS AND SYSTEMS, 45(1992) 305-312. 1992

http://dx.doi.org/10.1016/0165-0114(92)90148-W

\section{in proceedings and in edited volumes}

A35-c11 Takashi Hasuike, Hideki Katagiri, Sensitivity Analysis for Portfolio Selection Problem Considering Investor's Subjectivity, The International MultiConference of Engineers and Computer Scientists 2010, Hong Kong, China, pp. 2186-2190. 2010

http://www.iaeng.org/publication/IMECS2010/IMECS2010_pp2186-2190.pdf

A35-c10 H.-J.Zimmermann, Fuzzy Mathematical Programming, in: Tomas Gal and Harvey J. Greenberg eds., Advances in Sensitivity Analysis and Parametric Programming, Kluwer Academic Publishers, 1997 1-40. 1997

A35-c9 Yozo Nakahara and Mitsuo Gen, CONSTRAINTS WITH INTERVAL COEFFICIENTS AND STRICTNESS PROBABILITY INDEX, in: Proceedings of the IEEE World Congress on Fuzzy Systems, pp. 1883-1888. 1994

http://ieeexplore.ieee.org/iel2/1124/8016/x0235120.pdf

(f) By application of Fullér's approach [A35], we have (page 1885)

A35-c8 Ping-Teng Chang, E. Stanley Lee, Fuzzy Decision Making: A Survey, in: P-Z Wang, K-F Loe eds., Between Mind and Computer: Fuzzy Science and Engineering, Advances in Fuzzy Systems Applications and Theory, vol. 1, World Scientific, [ISBN 978-981-02-1305-3], pp. 139-182. 1994

A35-c7 H.-J. Zimmermann, Methods and applications of fuzzy mathematical programming, in: R. R. Yager and L.A.Zadeh eds., An introduction to Fuzzy Logic Applications in intelligent Systems, Kluwer Academic Publishers, Boston, 1992 97-120. 1992

A35-c6 V.A.Rybkin and A.V.Yazenin, Regularization and stability of possibilistic linear programming problems, in: Proceedings of the Sixth European Congress on Intelligent Techniques and Soft Computing (EUFIT'98), Aachen, September 7-10, 1998, Verlag Mainz, Aachen, Vol. I, 1998 3741. 1998

A35-c5 Alexander V. Yazenin, Vladimir A. Rybkin, Strong and Weak Stability in Possibilistic Linear Programming, in: Proceedings of the Seventh European Congress on Intelligent Techniques and Soft Computing (EUFIT'99), Aachen, September 13-16, 1999 Verlag Mainz, Aachen, [ISBN 389653-808-X], 4 pages, (Proceedings on CD-Rom). 1999

\section{in books}


A35-c4 Boading Liu, Theory and Practice of Uncertain Programming Series: Studies in Fuzziness and Soft Computing, Vol. 102 2002, Springer, [ISBN: 978-3-7908-1490-3] 2002.

A35-c3 Y.J.Lai and C.L.Hwang, Fuzzy Multiple Objective Decision Making, Lecture Notes in Economics and Mathematical Systems, No. 404, Springer Verlag, [ISBN: 978-3-540-57595-5], Berlin 1994.

A35-c2 Y.J.Lai and C.L.Hwang, Fuzzy Mathematical Programming, Methods and Applications, Lecture Notes in Economics and Mathematical Systems, No. 394, Springer Verlag, [ISBN 3-540-56098X], Berlin 1992.

A35-c1 R. Lowen, Fuzzy Set Theory, Kluwer Academic Publishers, [ISBN: 0-7923-4057-4], 1996. 